\title{
Low-Frequency Model-Order Reduction of Electromagnetic Fields Without Matrix Factorization
}

\author{
Rob F. Remis
}

\begin{abstract}
In this paper, we develop a reduced-order modeling technique, which is based on a low-frequency expansion of the electromagnetic field. The expansion can be written in terms of the pseudoinverse of a so-called system matrix. This pseudoinverse is given explicitly, and it is shown that it satisfies a reciprocity relation. Moreover, we show that computing matrix-vector products with this pseudoinverse essentially amounts to repeatedly solving Poisson's equation. The latter two properties allow us to efficiently compute reduced-order models via a Lanczos-type algorithm. The proposed method is illustrated by a number of numerical examples.
\end{abstract}

Index Terms-Electromagnetic fields, Lanczos-type algorithms, low-frequency expansion, model-order reduction.

\section{INTRODUCTION}

$\mathbf{M}$ ODEL-ORDER reduction enables us to compute approximate solutions of semidiscrete electromagnetic systems on a frequency interval of interest essentially at the cost of a single frequency (e.g., see [1] and [2]). Such systems typically have a large number of unknowns. For example, the order of the semidiscrete Maxwell system that arises after discretizing Maxwell's equations in space using a finite-difference or finite-element method easily runs into the millions for three-dimensional problems. Even one-dimensional problems may have hundreds or thousands of unknowns. The idea behind model-order reduction is to replace the large system by a much smaller one such that the solution of the smaller system approximates the solution of the large system on a frequency interval of interest.

The starting point of a model-order reduction method is the expansion of a frequency-response function around a certain expansion point. If a finite expansion point is taken then, in general, the factorization of a large matrix is required. Examples of methods that follow this approach are given in [3]-[6]. The factorization needs to be computed only once, but its computational costs are high and the factorization matrices need to be stored as well. As pointed out in [2], the factorization is undesirable if not prohibitive.

One way to avoid the computation of a matrix factorization is to take the expansion point to infinity. The resulting expansion

Manuscript received December 26, 2003; revised June 2, 2004.

The author is with the Laboratory of Electromagnetic Research, Delft University of Technology, 2600 GA Delft, The Netherlands.

Digital Object Identifier 10.1109/TMTT.2004.834577 is often called a high-frequency or early-time expansion and no matrix factorization is required in this case. The drawback is, however, that the order of the reduced-order model may become very large, especially if low frequencies are of interest (see [7] for a discussion and a two-step solution to this problem).

In this paper, it is shown that no matrix factorization needs to be computed (and, consequently, no factorization matrices need to be stored) if frequency zero is taken as an expansion point. More precisely, it is shown that the electromagnetic-field quantities can be expanded in terms of the pseudoinverse of a so-called system matrix. An explicit expression for this inverse is presented and some of its properties are discussed. In particular, we show that the pseudoinverse satisfies reciprocity and that its action on a vector can be computed efficiently since it amounts to solving Poisson's equation twice. The latter two properties allow the construction of low-frequency reduced-order models in an efficient manner.

This paper is inspired by the results presented in [8], where a low-frequency expansion of three-dimensional diffusive electromagnetic fields (displacement current is neglected in Maxwell's equations) is constructed. Here, the full Maxwell wave equations are considered and do not neglect the displacement currents. However, in this analysis, we restrict ourselves to one-dimensional configurations, not only because of simplicity, but also because, for one-dimensional configurations, Maxwell's equations are very similar to transmission-line equations and, consequently, the method proposed in this paper can be used to model (multiconductor) transmission lines as well. Furthermore, this formulation is such that the method is not restricted to one-dimensional problems only. Everything in this paper can be generalized to two and three dimensions.

This paper is organized as follows. In Section II, the semidiscrete Maxwell system is introduced and this system is written in terms of a so-called system matrix. A number of properties of this matrix are discussed in the Appendix. Subsequently, the electromagnetic field is expanded around zero frequency in terms of the pseudoinverse of the system matrix. The construction of the reduced-order models is based on this expansion. These models can be computed via a Lanczos-type algorithm since the pseudoinverse of the system matrix satisfies a reciprocity relation. The construction of the models and a brief description of the Lanczos algorithm can be found in Section III, and numerical results are presented in Section IV. Finally, $\|\cdot\|_{2}$ denotes the vector two-norm. 


\section{SEMIDISCRETE MaXWELl System}

We consider a one-dimensional electromagnetic field that satisfies the equations

$$
\begin{aligned}
\partial_{z} H_{y}+\sigma E_{x}+s \varepsilon E_{x} & =-J_{x}^{\mathrm{ext}} \\
\partial_{z} E_{x}+s \mu_{0} H_{y} & =0
\end{aligned}
$$

on $\left(0, \ell_{z}\right) \times \mathbb{C}^{+}$with $\ell_{z}>0$, and where $\mathbb{C}^{+}=\{s \in \mathbb{C} ; \operatorname{Re}(s) \geq$ $0\}$ denotes the right half of the complex $s$-plane. Before proceeding, let us first normalize these equations. Let $L>0$ denote a reference length and introduce the normalized coordinates

$$
z^{\prime}=L^{-1} z \text { and } s^{\prime}=L c_{0}^{-1} s
$$

where $c_{0}$ is the electromagnetic-wave speed in vacuum. In addition, we introduce the normalized field quantities

$$
\begin{aligned}
E_{x}^{\prime}\left(z^{\prime}, s^{\prime}\right) & =E_{x}\left(L z^{\prime}, c_{0} L^{-1} s^{\prime}\right) \\
H_{y}^{\prime}\left(z^{\prime}, s^{\prime}\right) & =Z_{0} H_{y}\left(L z^{\prime}, c_{0} L^{-1} s^{\prime}\right) \\
J_{x}^{\text {ext }}\left(z^{\prime}, s^{\prime}\right) & =Z_{0} L J_{x}^{\text {ext }}\left(L z^{\prime}, c_{0} L^{-1} s^{\prime}\right)
\end{aligned}
$$

and the normalized medium parameters

$$
\sigma^{\prime}\left(z^{\prime}\right)=Z_{0} L \sigma\left(L z^{\prime}\right) \text { and } \varepsilon_{r}\left(z^{\prime}\right)=\varepsilon_{r}\left(L z^{\prime}\right) .
$$

As is easily verified, these normalized quantities satisfy the equations

$$
\begin{aligned}
\partial_{z} H_{y}+\sigma E_{x}+s \varepsilon_{r} E_{x} & =-J_{x}^{\mathrm{ext}} \\
\partial_{z} E_{x}+s H_{y} & =0
\end{aligned}
$$

on $(0, \ell) \times \mathbb{C}^{+}$with $\ell=\ell_{z} / L$, and where we have dropped the primes. Finally, the computational domain is terminated by perfect electrically conducting (PEC) material boundary conditions given by

$$
\lim _{z \downarrow 0} E_{x}(z, s)=0 \text { and } \lim _{z \uparrow \ell} E_{x}(z, s)=0, \quad \text { for } s \in \mathbb{C}^{+} .
$$

We compute finite-difference approximations of the field quantities $E_{x}$ and $H_{y}$ on a nonuniform grid. To demonstrate certain symmetry properties of the finite-difference operators and to show our notation, we briefly review the standard finite-difference discretization of Maxwell's equations (e.g., see [9] and [10]). We begin by introducing primary and dual nodes. The primary nodes are given by $z_{r}=z_{r-1}+\delta_{z ; r}$ for $r=1,2, \ldots, R+1$, with $z_{0}=0, z_{R+1}=\ell$, and where all step sizes $\delta_{z ; r}$ are positive. The dual nodes are given by $\hat{z}_{r+1}=\hat{z}_{r}+\hat{\delta}_{z ; r}$ for $r=1,2, \ldots, R$ with $\hat{\delta}_{z ; r}>0$ for all $r$. Only staggered grids are considered for which the dual nodes interlace with the primary nodes.

The finite-difference approximations of $E_{x}$ and $H_{y}$ are denoted by $e_{x}$ and $h_{y}$ and satisfy the finite-difference equations

$$
\begin{aligned}
\frac{h_{y}\left(\hat{z}_{r+1}, s\right)-h_{y}\left(\hat{z}_{r}, s\right)}{\hat{\delta}_{z ; r}}+\sigma\left(z_{r}\right) e_{x}\left(z_{r}, s\right) & +s \varepsilon_{r}\left(z_{r}, s\right) \\
& =-j_{x}^{\operatorname{ext}}\left(z_{r}, s\right)
\end{aligned}
$$

for $r=1,2, \ldots, R$, and

$$
\frac{e_{x}\left(z_{r}, s\right)-e_{x}\left(z_{r-1}, s\right)}{\delta_{z ; r}}+s h_{y}\left(\hat{z}_{r}, s\right)=0
$$

for $r=1,2, \ldots, R+1$. In (1), $j_{x}^{\text {ext }}$ is a finite-difference approximation of $J_{x}^{\text {ext }}$. The PEC material boundary conditions are

$$
e_{x}\left(z_{0}, s\right)=0 \text { and } e_{x}\left(z_{R+1}, s\right)=0, \quad \text { for } s \in \mathbb{C}^{+} .
$$

Notice that this leaves us with $R$ unknowns for the electric-field strength, and $R+1$ unknowns for the magnetic-field strength, making a total of $2 R+1$ unknowns.

Introducing the vectors

$$
\begin{aligned}
\mathbf{f}_{e} & =\left[e_{x}\left(z_{1}, s\right), e_{x}\left(z_{2}, s\right), \ldots, e_{x}\left(z_{R}, s\right)\right]^{T} \\
\mathbf{f}_{h} & =\left[h_{y}\left(\hat{z}_{1}, s\right), h_{y}\left(\hat{z}_{2}, s\right), \ldots, h_{y}\left(\hat{z}_{R+1}, s\right)\right]^{T} \\
\mathbf{j}_{x}^{\text {ext }} & =\left[j_{x}^{\operatorname{ext}}\left(z_{1}, s\right), j_{x}^{\operatorname{ext}}\left(z_{2}, s\right), \ldots, j_{x}^{\operatorname{ext}}\left(z_{R}, s\right)\right]^{T}
\end{aligned}
$$

the finite-difference equations, including the PEC material boundary conditions, can be written as

$$
(D+S+s M) \mathbf{f}=-\mathbf{q}
$$

where the differentiation matrix is given by

$$
D=\left(\begin{array}{cc}
0 & \hat{Z} \\
Z & 0
\end{array}\right)
$$

with

$$
\hat{Z}=\hat{W}_{z}^{-1} \operatorname{bidiag}(-1,1) \text { and } Z=-W_{z}^{-1}[\operatorname{bidiag}(-1,1)]^{T}
$$

and the matrices $\hat{W}_{z}$ and $W_{z}$ are given by

$$
\begin{aligned}
& \hat{W}_{z}=\operatorname{diag}\left(\hat{\delta}_{z ; 1}, \hat{\delta}_{z ; 2}, \ldots, \hat{\delta}_{z ; R}\right) \\
& W_{z}=\operatorname{diag}\left(\delta_{z ; 1}, \delta_{z ; 2}, \ldots, \delta_{z ; R+1}\right) .
\end{aligned}
$$

Furthermore, $\operatorname{bidiag}(-1,1)$ is $R$-by- $(R+1)$ and upper bidiagonal with -1 on the diagonal and +1 on the upper diagonal. An obvious, but important symmetry relation is

$$
\hat{Z}^{T} \hat{W}_{z}=-W_{z} Z \text {. }
$$

Returning to (3), the medium matrices are given by

$$
S=\left(\begin{array}{cc}
M_{\sigma} & 0 \\
0 & 0
\end{array}\right) \text { and } M=\left(\begin{array}{cc}
M_{\varepsilon} & 0 \\
0 & I_{R+1}
\end{array}\right)
$$

where $I_{R+1}$ is the identity matrix of order $R+1$, and

$$
M_{\sigma}=\operatorname{diag}\left(\sigma\left(z_{1}\right), \sigma\left(z_{2}\right), \ldots, \sigma\left(z_{R}\right)\right)
$$

while

$$
M_{\varepsilon}=\operatorname{diag}\left(\varepsilon\left(z_{1}\right), \varepsilon\left(z_{2}\right), \ldots, \varepsilon\left(z_{R}\right)\right) .
$$

Finally, the field and source vector are given by

$$
\mathbf{f}=\left(\begin{array}{c}
\mathbf{f}_{e} \\
\mathbf{f}_{h}
\end{array}\right) \text { and } \mathbf{q}=\left(\begin{array}{c}
\mathbf{j}_{x}^{\text {ext }} \\
\mathbf{0}
\end{array}\right)
$$

respectively.

As a next step, we rewrite (3) in a more convenient form. First, let us introduce the diagonal matrix

$$
W=\left(\begin{array}{cc}
\hat{W}_{z} & 0 \\
0 & W_{z}
\end{array}\right)
$$


This matrix and the medium matrix $M$ are both diagonal and positive definite so that we can rewrite (3) in the form

$$
(A+s I) \tilde{\mathbf{f}}=-\tilde{\mathbf{q}}
$$

where $I$ is the identity matrix of order $2 R+1$, and $A$ is the system matrix given by

$$
A=M^{-1 / 2} W^{1 / 2}(D+S) W^{-1 / 2} M^{-1 / 2} .
$$

The scaled field and source vector are given by

$$
\tilde{\mathbf{f}}=M^{1 / 2} W^{1 / 2} \mathbf{f} \text { and } \tilde{\mathbf{q}}=M^{-1 / 2} W^{1 / 2} \mathbf{q}
$$

respectively. Some important properties of the system matrix are discussed in the Appendix.

In most practical cases, the $s$-dependence of the external current source can be factored out, i.e., $\mathbf{j}_{x}^{\text {ext }}$ can be written as

$$
\mathbf{j}_{x}^{\text {ext }}=w(s) \mathbf{j}
$$

where $\mathbf{j}$ is an $s$-independent vector. The scalar function $w$ is called the source wavelet or source signature. For source vectors of the above form, the scaled source vector $\tilde{\mathbf{q}}$ is given by

$$
\tilde{\mathbf{q}}=w(s) M^{-1 / 2} W^{1 / 2}\left(\begin{array}{l}
\mathbf{j} \\
\mathbf{0}
\end{array}\right)=w(s) \mathbf{p}
$$

where vector $\mathbf{p}$ is implicitly defined in the above equation. Equation (5) now becomes

$$
(A+s I) \tilde{\mathbf{f}}=-w(s) \mathbf{p} .
$$

For $s=0$, the above equation simplifies to

$$
A \tilde{\mathbf{f}}=-w(0) \mathbf{p} .
$$

In the Appendix, it is shown that the system matrix is singular. This implies that (8) does not have a solution or there are infinitely many solutions. No solution exists if vector $\mathbf{p}$ is outside the range of the system matrix. However, given the particular form of vector $\mathbf{p}$, we can show that this vector is in the range of the system matrix. One way of showing this is the following. Let $A^{\dagger}$ denote the pseudoinverse of matrix $A$ and recall that $A A^{\dagger}$ is the orthogonal projection onto the range of matrix $A$. Using the expression for the pseudoinverse, as given in the Appendix, it is easily verified that

$$
A A^{\dagger} \mathbf{p}=\mathbf{p} .
$$

Obviously, vector $\mathbf{p}$ is in the range of matrix $A$ and we conclude that (8) has infinitely many solutions. Of all these solutions, we take the minimum norm solution, which is given by

$$
\tilde{\mathbf{f}}=-w(0) A^{\dagger} \mathbf{p} .
$$

The inverse of $A+s I$ exists for nonzero $s \in \mathbb{C}^{+}$and (7) can be written as

$$
\tilde{\mathbf{f}}=-w(s)(A+s I)^{-1} \mathbf{p} .
$$

With (9), this can be written as

$$
\tilde{\mathbf{f}}=-w(s)(A+s I)^{-1} A A^{\dagger} \mathbf{p}
$$

and after a little manipulation, we obtain

$$
\tilde{\mathbf{f}}=-w(s) A^{\dagger}\left(I+s A^{\dagger}\right)^{-1} \mathbf{p}
$$

which holds for $s \in \mathbb{C}^{+} \backslash\{0\}$. For $s=0$, the above expression for the scaled field vector can be taken as the solution of (8) as well since (11) reduces to the minimum norm solution of (10) for this particular value of $s$.

Having found the scaled field vector in terms of the pseudoinverse of the system matrix, we can expand this vector around $s=0$ to obtain

$$
\begin{aligned}
\tilde{\mathbf{f}} & =-w(s) A^{\dagger} \sum_{k=0}^{\infty}\left(-s A^{\dagger}\right)^{k} \mathbf{p} \\
& =-w(s) A^{\dagger}\left[I-s A^{\dagger}+s^{2}\left(A^{\dagger}\right)^{2}-\cdots\right] \mathbf{p} .
\end{aligned}
$$

This is the low-frequency expansion we are looking for. In analogy with continuous problems, we call it the Rayleigh series for semidiscrete problems (see [11]) and it serves as a basis for the construction of the reduced-order models, which we describe in Section III.

\section{REDUCED-ORDER MODELS}

The construction of the reduced-order models is based on a Lanczos-type algorithm. This algorithm can be derived from the standard nonsymmetric Lanczos algorithm (e.g., see [12]) by exploiting the reciprocity relation (see the Appendix)

$$
\left(A^{\dagger}\right)^{T} \Delta^{-}=\Delta^{-} A^{\dagger}
$$

and by making a suitable choice for one of the starting vectors in the nonsymmetric Lanczos algorithm. Details of this procedure are given in [13] and [14] (see also [15]). The resulting algorithm is as follows.

\section{Reciprocity Based Lanczos-Type Algorithm}

1) Given the starting vector $\mathbf{v}_{1}=\|\mathbf{p}\|_{2}^{-1} \mathbf{p}$,

2) Set $\mathbf{v}_{0}=\mathbf{0}$ and $\gamma_{1}=\delta_{0}=1$,

3) For $k=1,2, \ldots$, compute

$$
\begin{aligned}
\delta_{k} & =\mathbf{v}_{k}^{T} \Delta^{-} \mathbf{v}_{k} \\
\alpha_{k} & =\delta_{k}^{-1} \mathbf{v}_{k}^{T} \Delta^{-} A^{\dagger} \mathbf{v}_{k} \\
\tilde{\mathbf{v}}_{k+1} & =A^{\dagger} \mathbf{v}_{k}-\alpha_{k} \mathbf{v}_{k}-\delta_{k} \delta_{k-1}^{-1} \gamma_{k} \mathbf{v}_{k-1} \\
\gamma_{k+1} & =\left\|\tilde{\mathbf{v}}_{k+1}\right\|_{2} \\
\mathbf{v}_{k+1} & =\gamma_{k+1}^{-1} \tilde{\mathbf{v}}_{k+1}
\end{aligned}
$$

end for

Some remarks about this algorithm. First, observe that the pseudoinverse appears in a matrix-vector product only. In the Appendix, it is shown that computing this product amounts to solving Poisson's equation twice. Efficient Poisson solvers are available for this purpose (e.g., see [16]). Second, the algorithm cannot continue if $\gamma_{k}$ or $\delta_{k}$ vanishes since division by these coefficients is required. A vanishing $\gamma_{k}$ is a happy event since this indicates that $\tilde{\mathbf{v}}_{k}$ vanishes, which means that we have computed a basis of an $A^{\dagger}$-invariant subspace. This is referred to as a regular termination of the algorithm. Unfortunately, such terminations almost do not occur in practice. More severe is a van- 
ishing $\delta_{k}$ for a nonzero vector $\mathbf{v}_{k}$. The algorithm stops without having constructed a basis for an $A^{\dagger}$-invariant subspace. This is referred to as a breakdown of the algorithm. Just as bad are near breakdowns for which $\delta_{k} \approx 0$. Breakdowns may be avoided by so-called look-ahead techniques (see [17] and the references cited therein), but we do not discuss these techniques in this paper since we have never detected a breakdown of the algorithm in practice. We stress, however, that we cannot guarantee that no breakdowns will occur during the iteration process.

Assuming now that $m$ steps of the reciprocity-based Lanczos-type algorithm have been carried out successfully, we have

$$
A^{\dagger} V_{m}=V_{m} T_{m}+\tilde{\mathbf{v}}_{m+1} \mathbf{e}_{m}^{T}
$$

where the $n$-by- $m$ matrix $V_{m}$ is given by

$$
V_{m}=\left(\mathbf{v}_{1}, \mathbf{v}_{2}, \ldots, \mathbf{v}_{m}\right)
$$

and $\mathbf{e}_{m}$ is the $m$ th column of the $m$-by- $m$ identity matrix. Furthermore, matrix $T_{m}$ is a real and tridiagonal matrix of order $m$ given by

$$
T_{m}=\left(\begin{array}{cccccc}
\alpha_{1} & \frac{\delta_{2}}{\delta_{1}} \gamma_{2} & & & & \\
\gamma_{2} & \alpha_{2} & \frac{\delta_{3}}{\delta_{2}} \gamma_{3} & & & \\
& & & \cdot & & \\
& & & & \cdot & \frac{\delta_{m}}{\delta_{m-1}} \gamma_{m}
\end{array}\right) .
$$

Having the Lanczos decomposition of (13) at our disposal, it can be shown by induction that we also have (e.g., see [18])

$$
\left(A^{\dagger}\right)^{k} \mathbf{p}=\|\mathbf{p}\|_{2} V_{m} T_{m}^{k} \mathbf{e}_{1}
$$

for $k=0,1, \ldots, m-1$. Using this result in the low-frequency expansion given by (12), we can write the scaled field vector as

$$
\tilde{\mathbf{f}}(s)=\tilde{\mathbf{f}}_{m}(s)+\tilde{\mathbf{z}}_{m}(s)
$$

where we have introduced the reduced-order model

$$
\tilde{\mathbf{f}}_{m}(s)=-w(s)\|\mathbf{p}\|_{2} V_{m} T_{m}\left(I_{m}+s T_{m}\right)^{-1} \mathbf{e}_{1} .
$$

This model approximates the scaled field vector with an error given by

$$
\tilde{\mathbf{z}}_{m}(s)=-w(s) \sum_{k=m-1}^{\infty}(-s)^{k}\left[\left(A^{\dagger}\right)^{k+1} \mathbf{p}-\|\mathbf{p}\|_{2} V_{m} T_{m}^{k+1} \mathbf{e}_{1}\right] .
$$

Notice that the error vector vanishes for $s=0$ as it should, of course. Finally, matrix $T_{m}\left(I_{m}+s T_{m}\right)^{-1}$ is computed for all frequencies of interest by first computing the Schur decomposition of matrix $T_{m}$ and, subsequently, backsubstitution is used for each frequency.

\section{NUMERICAL RESULTS}

To illustrate the performance of the model-order reduction technique, we present some numerical examples. The first configuration consists of a homogeneous medium characterized by

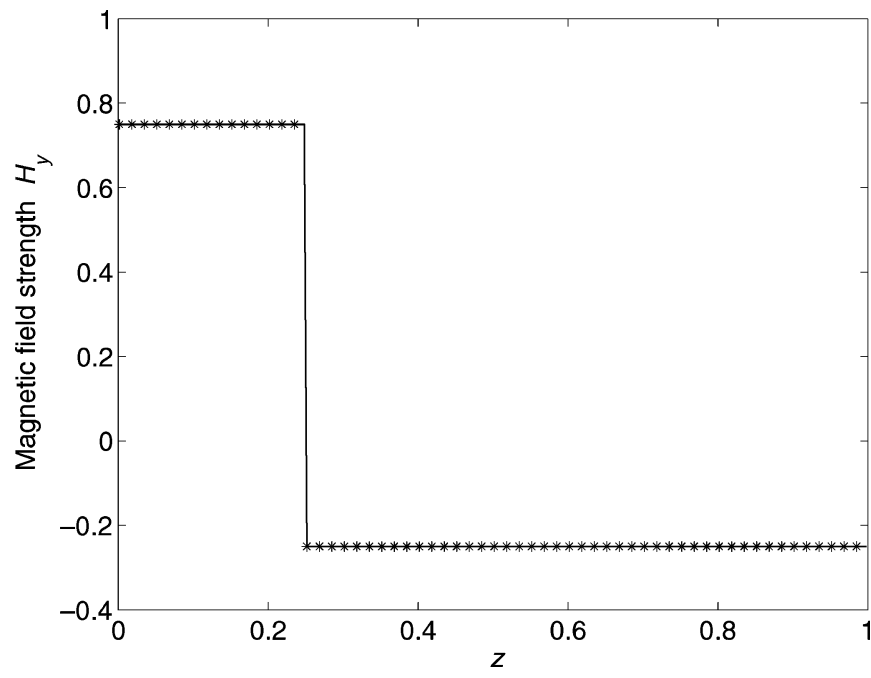

Fig. 1. Static minimum norm magnetic field. Source is located at $z_{s}=1 / 4$ Solid line signifies exact result. Symbols show the finite-difference approximation of (10).

a conductivity $\sigma=4 \mathrm{mS} / \mathrm{m}$, and a relative permittivity $\varepsilon_{r}=4$. We take $s=j \omega$, with $\omega=2 \pi f$, and consider frequencies in the range $0 \leq f \leq 10^{8} \mathrm{~Hz}$. We use a uniform grid with a step sizes such that we have 31 points per smallest wavelength $\lambda_{\min }$. The size of the total domain is $10 \lambda_{\min }$. We set the reference length $L$ equal to the domain size and take for the external current source a planar electric-current sheet represented by

$$
J_{x}^{\text {ext }}=w(s) \delta\left(z-z_{s}\right)
$$

where $z_{s}$ is the source location, and we set, without loss of generality, $w(s)=1$ for all frequencies of interest. The order of the system for this particular configuration is 599 .

Before showing any reduced-order models, let us first compute the minimum norm static field. As is easily verified, the static electric-field strength vanishes, and if the external electric-current source is located at $z_{s}=1 / 4$, the minimum norm static magnetic field is given by

$$
H_{y}= \begin{cases}\frac{3}{4}, & \text { if } 0<z<\frac{1}{4} \\ \frac{-1}{4}, & \text { if } \frac{1}{4}<z<1 .\end{cases}
$$

Computing now the minimum norm static field according to (10), we obtain the results shown in Fig. 1. Only the magneticfield strength is shown since the static electric-field strength vanishes. The minimum norm static field is computed exactly at the dual nodes of the grid.

To study the convergence behavior of the reduced-order models, we show in Fig. 2 the relative error

$$
E_{m}^{\mathrm{rel}}(s)=\frac{\left\|\mathbf{h}_{m}(s)-\mathbf{h}^{\text {exact }}(s)\right\|_{2}}{\left\|\mathbf{h}^{\text {exact }}(s)\right\|_{2}}
$$

as a function of frequency. Frequency zero is excluded from this figure since we have already seen that it is computed exactly. Furthermore, $\mathbf{h}_{m}$ is a vector containing all magnetic-field strength approximations of order $m$, and $\mathbf{h}^{\text {exact }}$ is a vector containing the exact magnetic-field strength values at the dual nodes of the grid. We observe that the magnetic-field strength 


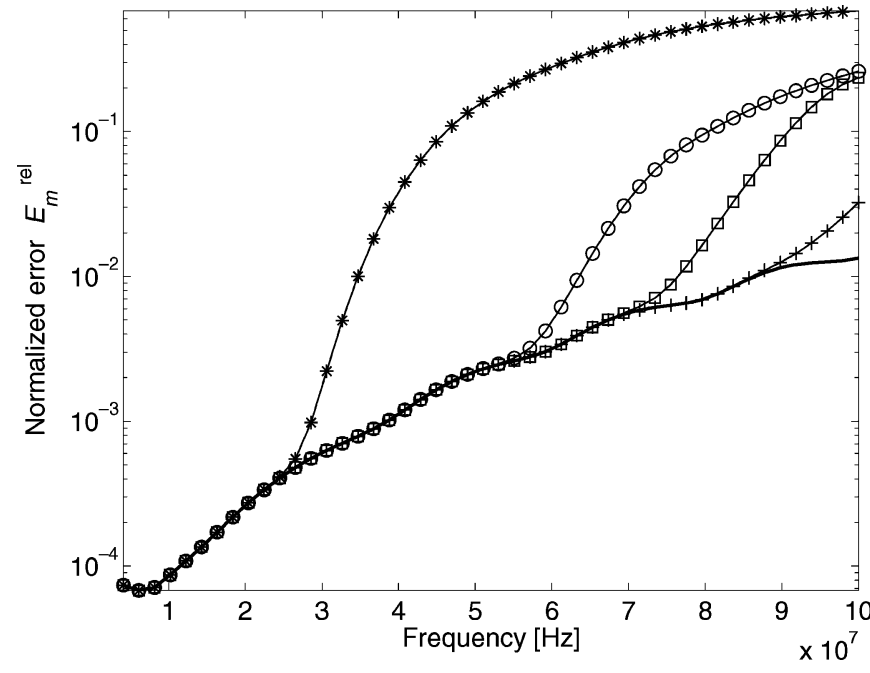

Fig. 2. Normalized error $E_{m}^{\mathrm{rel}}$ of (14) on the frequency interval of interest for models of order 20 (star), 40 (circle), 60 (square), 80 (plus), and 100 (solid line).

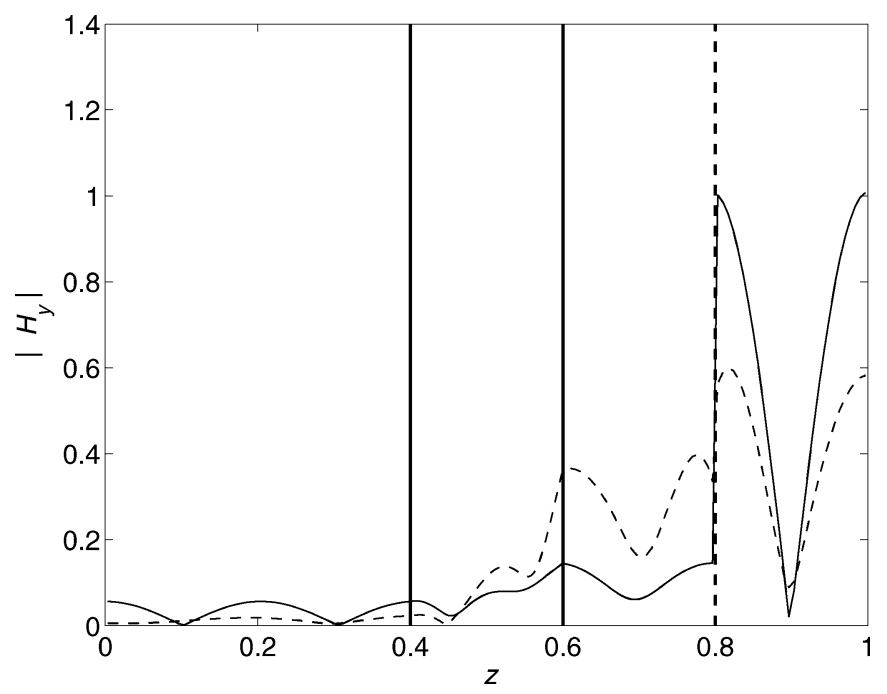

Fig. 3. Absolute value of the reduced-order models for the magnetic-field strength obtained after 15 iterations (dashed line), and 50 iterations (solid line) of the Lanczos algorithm. Frequency is $5 \cdot 10^{7} \mathrm{~Hz}$.

for low frequencies is approximated first. Also note that convergence slows down as frequency increases. No significant improvements were observed on this frequency interval after 100 Lanczos iterations.

In our second example, we consider the same frequency interval as in the previous example. The configuration consists of a vacuum domain in which a homogeneous slab is present. The slab has a relative permittivity $\varepsilon_{r}=4$ and a conductivity $\sigma=6 \mathrm{mS} / \mathrm{m}$. We use a nonuniform grid such that we locally have 31 points per smallest wavelength. This implies that the grid is refined by a factor 2 inside the slab since the wavelength is two times smaller in the slab compared with the wavelength in the surrounding vacuum domain. A dual node is placed halfway two primary nodes, i.e.,

$$
\hat{z}_{r}=z_{r-1}+\frac{\delta_{z ; r}}{2}, \quad \text { for } r=1,2, \ldots, R+1 .
$$

The size of the total domain is $10 \lambda_{\min }$, where $\lambda_{\min }$ is the smallest wavelength in the slab. Again, we set the reference

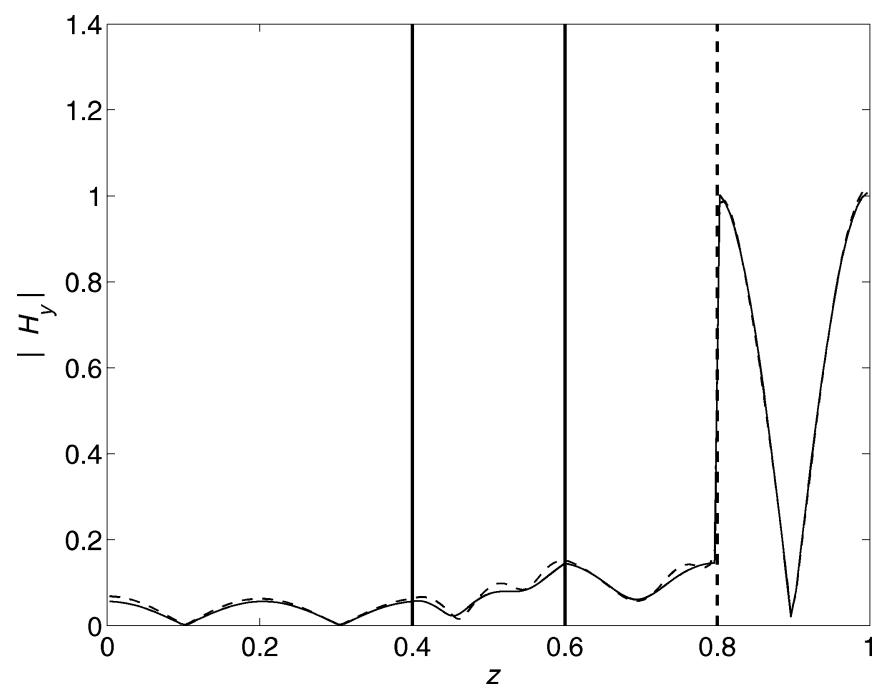

Fig. 4. Absolute value of the reduced-order models for the magnetic-field strength obtained after 25 iterations (dashed line), and 50 iterations (solid line) of the Lanczos algorithm. Frequency is $5 \cdot 10^{7} \mathrm{~Hz}$.

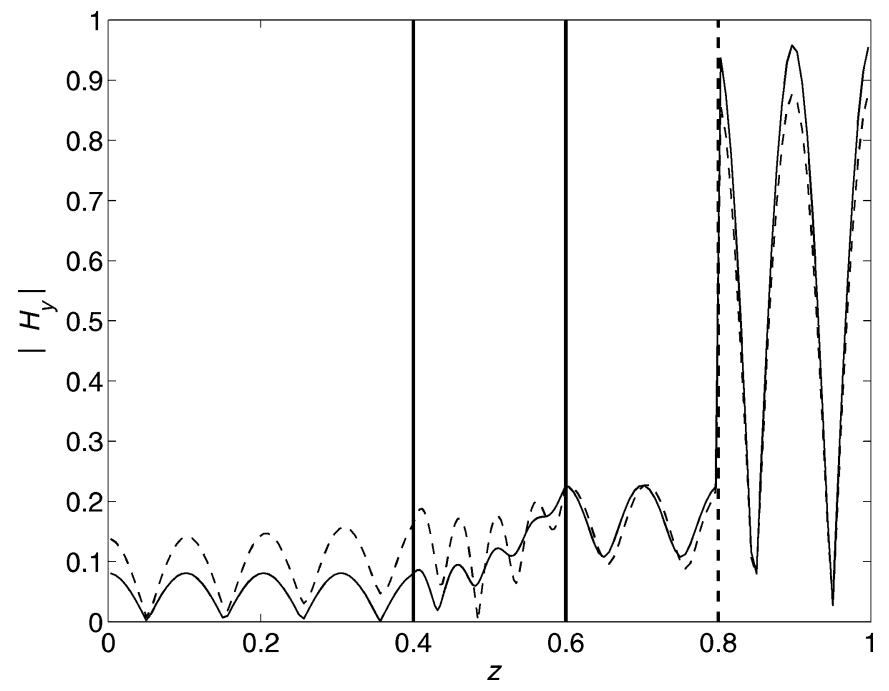

Fig. 5. Absolute value of the reduced-order models for the magnetic-field strength obtained after 50 iterations (dashed line), and 80 iterations (solid line) of the Lanczos algorithm. Frequency is $10^{8} \mathrm{~Hz}$.

length equal to the domain size. In normalized coordinates, the slab occupies the domain $0.4<z<0.6$, and the electric-current source is the same as in the previous example, except that it is located at $z_{s}=0.8$. The order of the system for this particular configuration is 359 .

In Figs. 3 and 4, we show the absolute value of the normalized magnetic-field strength on the total computational domain at a frequency of $5 \cdot 10^{7} \mathrm{~Hz}$. The solid vertical lines in these figures show where the slab is located, while the dashed vertical line indicates the location of the planar current sheet. The dashed line in Fig. 3 shows the reduced-order model obtained after 15 iterations and, in Fig. 4, after 25 iterations of the Lanczos-type algorithm. The solid line in these figures is the reduced-order model obtained after 50 iterations. We observe that the results improve as the number of iterations increases. Also note that the magnetic-field strength jumps across the source location. 


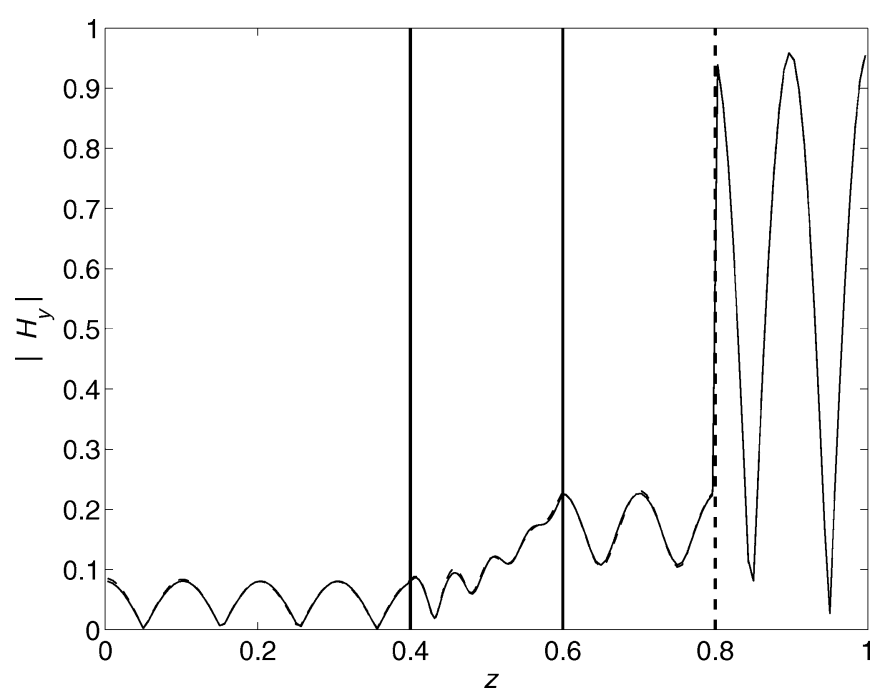

Fig. 6. Absolute value of the reduced-order models for the magnetic-field strength obtained after 70 iterations (dashed line), and 80 iterations (solid line) of the Lanczos algorithm. Frequency is $10^{8} \mathrm{~Hz}$.

If we now fix the order of the model to 50 and increase the frequency to $10^{8} \mathrm{~Hz}$, we obtain the results shown in Fig. 5 . The dashed line in this figure shows the reduced-order model of order 50, while the solid line is the reduced-order model obtained after 80 iterations. Increasing the order of the model from 50 to 70, we arrive at the results shown in Fig. 6. It is clear that more iterations are required to obtain reliable results at higher frequencies. Fifty iterations are sufficient for a frequency of $5 \cdot 10^{7} \mathrm{~Hz}$, but insufficient for a frequency of $10^{8} \mathrm{~Hz}$.

\section{CONClusions}

In this paper, we have described a reduced-order modeling technique, which is based on a low-frequency expansion of the electromagnetic field. We showed that this expansion can be written in terms of the pseudoinverse of the system matrix and no matrix factorization is required.

The system matrix is singular because PEC material boundary conditions are imposed at the boundary of the computational domain. If a problem with a PEC material boundary condition at one end, and a perfect magnetically conducting boundary condition (PMC material boundary condition) at the other end is considered, it can be shown that the system matrix is no longer singular. For a problem with this type of boundary conditions, the inverse of the system matrix can be written in terms of inverse Laplace operators as well and the analysis is essentially the same as the analysis presented in this paper. Furthermore, we can analyze a configuration with PMC material boundary conditions at both ends of the domain by simply reversing the roles of the electric- and magnetic-field strength in our formulation.

Future research will focus on the extension of the method to two- and three-dimensional configurations. Loosely speaking, the only problem is to find the (pseudo) inverse of the system matrix in these cases since the construction of the low-frequency expansion is essentially the same as the one presented in this paper.

\section{APPENDIX}

PROPERTIES OF THE SYSTEM MATRIX

Property 1: The system matrix is skew symmetric for lossless media. Matrix $S$ vanishes for lossless media, and the system matrix simplifies to

$$
A=M^{-1 / 2} W^{1 / 2} D W^{-1 / 2} M^{-1 / 2} .
$$

Furthermore, from (4), it follows that

$$
D^{T} W=-W D
$$

which shows that the differentiation matrix is $W$-skew symmetric. Using (15), it is easily verified that the system matrix is skew symmetric.

Property 2: The system matrix is singular for lossless media. This property follows directly from the fact that $A$ is skew symmetric for lossless media and its order is odd. Another way of showing that matrix $A$ is singular is the following. First, observe that the null space of matrix $\hat{Z}$ is one-dimensional and spanned by the $(R+1)$-by- 1 vector $\mathbf{e}=[1,1, \ldots, 1]^{T}$. This should not be a surprise since the PEC material boundary conditions specify the static magnetic-field strength up to an additional constant. Now let

$$
\mathbf{u}_{0}=M^{1 / 2} W^{1 / 2}\left(\begin{array}{l}
\mathbf{0} \\
\mathbf{e}
\end{array}\right)
$$

where $\mathbf{0}$ is the $R$-by- 1 zero vector. Computing the matrix-vector product $A \mathbf{u}_{0}$ shows that this product vanishes, and since $\mathbf{u}_{0}$ does not, we conclude that matrix $A$ is singular for lossless media. Loosely speaking, the static magnetic field lives in the null space of the system matrix. For completeness, we mention that the null space of matrix $Z$ is trivial.

Property 3: The system matrix is singular for lossy media. For lossy media, the system matrix is given by (6). Let $\mathbf{u}_{0}$ be as above, and compute

$$
\begin{aligned}
A \mathbf{u}_{0} & =M^{-1 / 2} W^{1 / 2}(D+S) W^{-1 / 2} M^{-1 / 2} M^{1 / 2} W^{1 / 2}\left(\begin{array}{l}
\mathbf{0} \\
\mathbf{e}
\end{array}\right) \\
& =M^{-1 / 2} W^{1 / 2}\left[D\left(\begin{array}{l}
\mathbf{0} \\
\mathbf{e}
\end{array}\right)+S\left(\begin{array}{l}
\mathbf{0} \\
\mathbf{e}
\end{array}\right)\right] \\
& =\mathbf{0} .
\end{aligned}
$$

This shows that the system matrix is singular for lossy media as well.

Property 4: The pseudoinverse of the system matrix is given by

$$
A^{\dagger}=M^{1 / 2} W^{1 / 2} \tilde{D} W^{-1 / 2} M^{1 / 2}
$$

where

$$
\tilde{D}=\left(\begin{array}{cc}
0 & D_{2} \\
D_{1} & -D_{1} M_{\sigma} D_{2}
\end{array}\right)
$$

with

$$
D_{1}=Z(\hat{Z} Z)^{-1} \text { and } D_{2}=(\hat{Z} Z)^{-1} \hat{Z} \text {. }
$$

The proof consists of verifying the Moore-Penrose conditions (see [12]) using (4). Note that matrix $\hat{Z} Z$ is the discretized 
Laplacian with Dirichlet boundary conditions. This matrix satisfies the symmetry relation

$$
(\hat{Z} Z)^{T} \hat{W}_{z}=\hat{W}_{z}(\hat{Z} Z)
$$

and it can be shown that all its eigenvalues are simple and negative.

Property 5: Introduce the signature matrix

$$
\Delta^{-}=\left(\begin{array}{cc}
I_{R} & 0 \\
0 & -I_{R+1}
\end{array}\right) .
$$

The pseudoinverse satisfies the reciprocity relation

$$
\left(A^{\dagger}\right)^{T} \Delta^{-}=\Delta^{-} A^{\dagger}
$$

This property is straightforward to verify using (4) and (16).

Property 6: Computing the matrix-vector product $\mathbf{y}=A^{\dagger} \mathbf{x}$ for a given vector $\mathbf{x}$ essentially amounts to solving Poisson's equation twice.

To see this, first set $\tilde{\mathbf{x}}=W^{-1 / 2} M^{1 / 2} \mathbf{x}$ and $\tilde{\mathbf{y}}=\tilde{D} \tilde{\mathbf{x}}$, then $\mathbf{y}=M^{1 / 2} W^{1 / 2} \tilde{\mathbf{y}}$. The only nontrivial part is the computation of vector $\tilde{\mathbf{y}}$. Partition this vector and $\tilde{\mathbf{x}}$ in the same way as the field vector. Using the definition of matrix $\tilde{D}$, we obtain

$$
\begin{aligned}
\left(\begin{array}{c}
\tilde{\mathbf{y}}_{e} \\
\tilde{\mathbf{y}}_{h}
\end{array}\right) & =\left(\begin{array}{cc}
0 & D_{2} \\
D_{1} & -D_{1} M_{\sigma} D_{2}
\end{array}\right)\left(\begin{array}{c}
\tilde{\mathbf{x}}_{e} \\
\tilde{\mathbf{x}}_{h}
\end{array}\right) \\
& =\left(\begin{array}{c}
D_{2} \tilde{\mathbf{x}}_{h} \\
D_{1} \tilde{\mathbf{x}}_{e}-D_{1} M_{\sigma} D_{2} \tilde{\mathbf{x}}_{h}
\end{array}\right) .
\end{aligned}
$$

In other words, for $\tilde{\mathbf{y}}_{e}$, we have

$$
(\hat{Z} Z) \tilde{\mathbf{y}}_{e}=\hat{Z} \tilde{\mathbf{x}}_{h}
$$

which is Poisson's equation. Similarly, for $\tilde{\mathbf{y}}_{h}$, we have $\tilde{\mathbf{y}}_{h}=$ $Z \tilde{\mathbf{z}}$, where $\tilde{\mathbf{z}}$ satisfies

$$
(\hat{Z} Z) \tilde{\mathbf{z}}=\tilde{\mathbf{x}}_{e}-M_{\sigma} \tilde{\mathbf{y}}_{e}
$$

This is the second Poisson equation that needs to be solved.

\section{ACKNOWLEDGMENT}

The author would like to thank the anonymous reviewers for their constructive comments. The author also extends many thanks to N. Budko, Laboratory of Electromagnetic Research, Delft University, Delft, The Netherlands, for discussions and support.

\section{REFERENCES}

[1] P. Feldmann and R. W. Freund, "Efficient linear circuit analysis by Padé approximation via the Lanczos process," IEEE Trans. Computer-Aided Design, vol. 14, pp. 639-649, May 1995.

[2] A. C. Cangellaris, M. Celik, S. Pasha, and L. Zhao, "Electromagnetic model order reduction for system-level modeling," IEEE Trans. Microwave Theory Tech., vol. 47, pp. 840-850, June 1999.

[3] D. K. Sun, J. F. Lee, and Z. J. Cendes, "ALPS-A new fast frequency-sweep procedure for microwave devices," IEEE Trans. Microwave Theory Tech., vol. 49, pp. 398-402, Feb. 2001.

[4] J. E. Bracken, D. K. Su, and Z. J. Cendes, " $S$-domain methods for simultaneous time and frequency characterization of electromagnetic devices," IEEE Trans. Microwave Theory Tech., vol. 46, pp. 1277-1290, Sept. 1998

[5] Y. Zhu and A. C. Cangellaris, "Finite element-based model order reduction of electromagnetic devices," Int. J. Numer. Modeling, vol. 15, pp. 73-92, 2002.

[6] B. Denecker, F. Olyslager, L. Knockaert, and D. De Zutter, "Generation of FDTD subcell equations by means of reduced order modeling," IEEE Trans. Antennas Propagat., vol. 51, pp. 1806-1817, Aug. 2003.

[7] T. Wittig, I. Munteanu, R. Schuhmann, and T. Weiland, "Two-step Lanczos algorithm for model order reduction," IEEE Trans. Magn., vol. 38, pp. 673-676, Mar. 2002.

[8] V. L. Druskin, L. A. Knizhnerman, and P. Lee, "New spectral Lanczos decomposition method for induction modeling in arbitrary 3-D geometry," Geophysics, vol. 64, pp. 701-706, 1999.

[9] A. Taflove and S. C. Hagness, Computational Electrodynamics: The Finite-Difference Time-Domain Method, 2nd ed. Norwood, MA: Artech House, 2000.

[10] K. S. Yee, "Numerical solution of initial boundary value problems involving Maxwell's equations in isotropic media," IEEE Trans. Antennas Propagat., vol. AP-14, pp. 302-307, May 1966.

[11] R. E. Kleinman, "The Rayleigh region," Proc. IEEE, vol. 53, pp. 848-856, Aug. 1965

[12] G. H. Golub and C. F. Van Loan, Matrix Computations, 3rd ed. Baltimore, MD: The Johns Hopkins Univ. Press, 1996.

[13] R. W. Freund, "Lanczos-type algorithms for structured non-Hermitean eigenvalue problems," in Proc. Cornelius Lanczos Int. Centenary Conf., 1993, pp. 243-245.

[14] R. W. Freund and N. M. Nachtigal, "Software for simplified Lanczos and QMR algorithms," Appl. Numer. Math., vol. 19, pp. 319-341, 1995.

[15] K. C. Jea and D. M. Young, "On the simplification of generalized conjugate-gradient methods for nonsymmetrizable systems," Linear Algebra and Its Applicat., vol. 52/53, pp. 399-417, 1983.

[16] Y. Saad, Iterative Methods for Sparse Linear Systems, 2nd ed. Philadelphia, PA: SIAM, 2003.

[17] R. W. Freund, "The look-ahead Lanczos process for nonsymmetric matrices and its applications," in Proc. Cornelius Lanczos Int. Centenary Conf., 1993, pp. 33-47.

[18] V. L. Druskin and L. A. Knizhnerman, "Two polynomial methods of calculating functions of symmetric matrices," U.S.S.R. Comput. Math. Math. Phys., vol. 29, pp. 112-121, 1989.

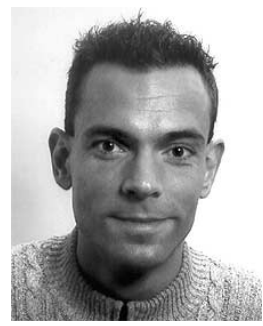

Rob F. Remis was born in Rotterdam, The Netherlands, on August 21, 1970. He received the M.Sc. and $\mathrm{Ph}$.D. degrees in electrical engineering from the Delft University of Technology, Delft, The Netherlands, in 1994 and 1998, respectively.

Since 1998, he has been an Assistant Professor with the Laboratory of Electromagnetic Research, Delft University of Technology. His personal research interest is in the field of computational electromagnetics. 